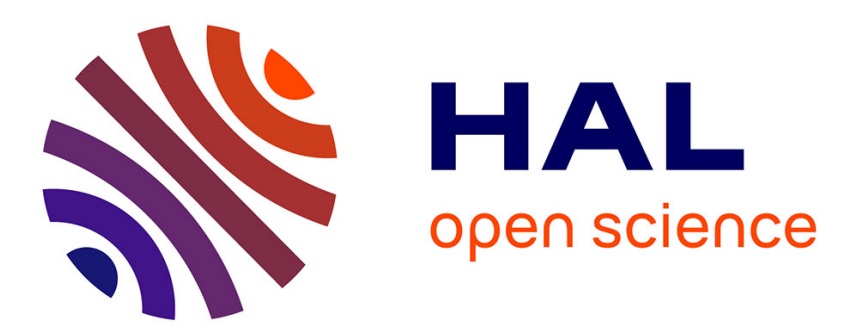

\title{
FEMs on composite meshes for plasma equilibrium simulations in tokamaks
}

\author{
Holger Heumann, Francesca Rapetti, Minh Duy Truong
}

\section{To cite this version:}

Holger Heumann, Francesca Rapetti, Minh Duy Truong. FEMs on composite meshes for plasma equilibrium simulations in tokamaks. 19th European Conference on Mathematics for Industry - ECMI 2016, Jun 2016, Santiago-de-Compostela Spain. hal-01397386

\section{HAL Id: hal-01397386 \\ https://hal.science/hal-01397386}

Submitted on 8 Dec 2016

HAL is a multi-disciplinary open access archive for the deposit and dissemination of scientific research documents, whether they are published or not. The documents may come from teaching and research institutions in France or abroad, or from public or private research centers.
L'archive ouverte pluridisciplinaire HAL, est destinée au dépôt et à la diffusion de documents scientifiques de niveau recherche, publiés ou non, émanant des établissements d'enseignement et de recherche français ou étrangers, des laboratoires publics ou privés. 


\title{
FEMs on composite meshes for plasma equilibrium simulations in tokamaks
}

\author{
Holger Heumann, Francesca Rapetti, Minh Duy Truong
}

\begin{abstract}
We rely on a combination of different finite element methods on composite meshes, for the simulation of axisymmetric plasma equilibria in tokamaks. One mesh with Cartesian quadrilaterals covers the vacuum chamber and one mesh with triangles discretizes the region outside the chamber. The two meshes overlap in a narrow region around the chamber. This approach gives the flexibility to achieve easily and at low cost higher order regularity for the approximation of the flux function in the area that is covered by the plasma, while preserving accurate meshing of the geometric details in the exterior. The continuity of the numerical solution across the boundary of each subdomain is enforced by a new mortar-like projection.
\end{abstract}

\section{Introduction}

The possibility of using composite meshes in finite element (FE) simulations of industrial problems is a recurrent topic $[12,14,7,4,13]$. Composite meshes are involved as soon as the global discretization of a PDE combines discretizations on local (overlapping or non-overlapping) subdomains, each suitably triangulated by non-matching grids. The reason for using composite meshes are various: fitting the geometry or the local smoothness of the solution, resolving multiple scales in

Holger Heumann

INRIA, CASTOR team, 2004 Route des Lucioles BP 9306902 Sophia Antipolis, France, e-mail: Holger.Heumann@inria.fr

Francesca Rapetti

Dept. of Mathematics, Univ. de Nice, Parc Valrose, 06108 Nice cedex 02, France, e-mail: Francesca.Rapetti@unice.fr

Minh Duy Truong

INRIA, CASTOR team, 2004 Route des Lucioles BP 9306902 Sophia Antipolis, France, e-mail: truongminhduy91@yahoo.com

The authors kindly acknowledge Inria for the financial support. 
regions with irregular data, using fast solvers on structured grids or a divide-andconquer/domain decomposition approach to very large problems on parallel machines.

In the present case, we are looking for a simple and practical approach to introduce in certain parts of the computational domain FE functions that are not only continuous, but have also first order, second order or higher order continuous derivatives. In general it is very difficult to introduce FE spaces over simplicial unstructured meshes with such properties. On the other hand, if we work with Cartesian meshes this becomes very simple. It is sufficient to use tensor products of spline spaces with sufficiently high regularity. So, as it is naive to expect that technical devices can be entirely triangulated with Cartesian meshes, we introduce composite meshes involving Cartesian meshes in those subdomains where we want high regular FE representations and triangular unstructured meshes in those subdomains where we want conformity with the geometry.

The industrial application we consider concerns the free boundary plasma equilibrium in tokamaks for nuclear fusion [2], mathematically described by the force balance and Maxwell's equations in the eddy-current approximation. By symmetry considerations, the free boundary plasma equilibrium problem can be reduced to a scalar semi-linear elliptic one for the poloidal flux. As the magnetic field and the current density are tangential to the level sets of the poloidal flux, the precise calculation of the level set distribution for the poloidal flux is fundamental in tokamak science. Hence, it is important to have good approximations not only of the poloidal flux but also of its derivatives.

In Figure 1 we show a sketch of the cross section of a tokamak. It contains the geometrical details such as coils, passive structures and the iron core, that need to be accurately resolved by a triangulation. A peculiarity of the free boundary plasma equilibrium problem is the unknown plasma domain, that is implicitly given as the domain that is bounded by the largest level set that it not intersected by the limiter. At the beginning, in tokamak devices the plasma was always attached to the limiter, later the focus shifted towards devices with so called divertors that allow to create plasmas that are completely detached from material. The boundary of such a plasma is characterized by saddle points, also called X-points, of the poloidal flux. The magnetic axis, located at the maximum of the poloidal flux, is another important critical point to be computed. So, here again, continuous higher derivatives of approximations of the poloidal flux, are very desirable.

Fig. 1 Right: Geometric description of the tokamak in the poloidal plane. Left and middle: Sketch for characteristic plasma shapes. The plasma boundary touches the limiter (middle) or the plasma is enclosed by a flux line that goes through an X-point (right).
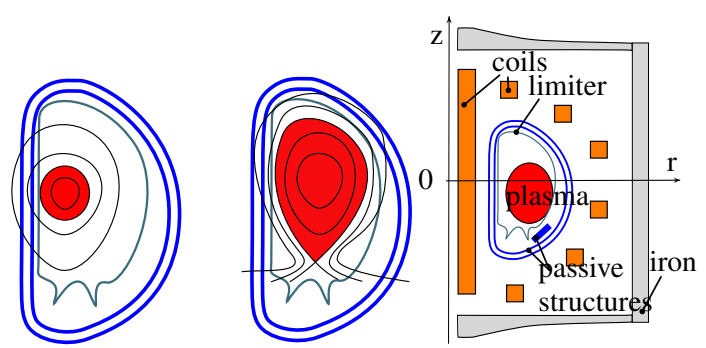
Fig. 2 Left: detail view for the WEST tokamak, with the iron core (green), the passive structures (red) and the various coils (light blue). Right: the composite meshes for the WEST tokamak.

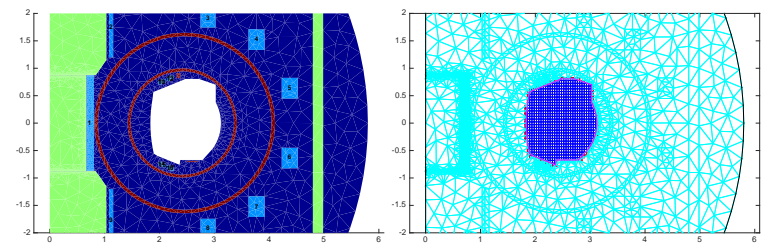

In [9], we showed that the numerical calculation of free boundary plasma equilibria highly benefits from approximating the poloidal flux through some higher regular FE functions in the interior of the limiter. In the present paper, we rather analyse the precision of the proposed approach, by varying the discretization parameters. We thus compute the approximation error between the computed and the synthetic solution of a model problem for the same method adopted in [9], by varying, for example, the local polynomial degree in the subdomains, the size of the overlap between the meshes, the local size of the mesh elements. Indeed, FE methods on composite meshes are widely used in practice, but their theoretical foundation is fairly limited in the literature. Therefore, we report here experimental convergence results for different discretization schemes involving composite meshes.

The outline is the following: In section 2 we recall the classical mortar element method (MEM) for overlapping meshes in order to introduce, in the following section 3, a modified method (MEM-M) that simplifies the implementation by avoiding integrals over cut elements. We then present experimental convergence results.

\section{The mortar element method (MEM) with overlapping meshes}

We consider the following Poisson problem for the unknown $\psi$ in the bounded domain $\Omega \subset \mathbb{R}^{n}$ with boundary $\Gamma=\partial \Omega$ :

$$
-\nabla \cdot(\nabla \psi)=f \quad \text { in } \Omega \quad \text { and } \quad \psi_{\mid \partial \Omega}=\psi_{0} \quad \text { in } \Gamma,
$$

where $\nabla$ (resp. $\nabla \cdot$ ) is the gradient (resp. divergence) operator in $\mathbb{R}^{n}$. The righthand side $f$ and the Dirichlet data $\psi_{0}$ are given. Let $L^{2}(\Omega)$, be the functional space of measurable functions on $\Omega$ that are square integrable in $\Omega$ and $H^{1}(\Omega)=$ $\left\{u \in L^{2}(\Omega), \nabla u \in L^{2}(\Omega)^{2}\right\}$ the Hilbert space endowed with the norm $\|u\|_{H^{1}(\Omega)}^{2}=$ $\|u\|_{\Omega}^{2}+|u|_{H^{1}(\Omega)}^{2}$ where $|u|_{H^{1}(\Omega)}^{2}=\|\nabla u\|_{\Omega}^{2}$. Let $\Omega^{\text {in }} \subset \Omega$ be a subdomain with $\Omega^{\text {in }} \cap \Gamma=\emptyset$ and $\Omega^{\text {ex }}=\Omega \backslash \Omega^{\text {in }}$ the complement of $\Omega^{\text {in }}$ in $\Omega$. Further, the boundary of $\Omega^{\text {in }}, \gamma:=\partial \Omega^{\text {in }}$, is the interface between $\Omega^{\text {ex }}$ and $\Omega^{\text {in }}$. To formulate (1) as a variational problem in a domain decomposition framework, let us introduce the functional space

$$
\mathscr{H}_{g}=\left\{(v, w) \in H^{1}\left(\Omega^{\mathrm{ex}}\right) \times H^{1}\left(\Omega^{\mathrm{in}}\right), v_{\mid \Gamma}=g, v_{\mid \gamma}=w_{\mid \gamma}\right\} .
$$

Then, the weak formulation of (1) is: Find $\left(\psi^{\mathrm{ex}}, \psi^{\mathrm{in}}\right) \in \mathscr{H}_{\psi_{0}}$ s.t. for all $(v, w) \in \mathscr{H}_{0}$ 


$$
\int_{\Omega^{\mathrm{ex}}} \nabla \psi^{\mathrm{ex}} \cdot \nabla v \mathrm{~d} \mathbf{x}+\int_{\Omega^{\mathrm{in}}} \nabla \psi^{\mathrm{in}} \cdot \nabla w \mathrm{~d} \mathbf{x}=\int_{\Omega^{\mathrm{ex}}} f v \mathrm{~d} \mathbf{x}+\int_{\Omega^{\mathrm{in}}} f w \mathrm{~d} \mathbf{x} .
$$

We wish to introduce different types of meshes $\mathscr{T}^{\text {ex }}$ and $\mathscr{T}^{\text {in }}$ in the two subdomains $\Omega^{\mathrm{ex}}$ and $\Omega^{\text {in }}$. To achieve a maximum of flexibility we do not expect the meshes $\mathscr{T}^{\mathrm{ex}}$ and $\mathscr{T}^{\text {in }}$ to be conforming with $\Omega^{\mathrm{ex}}$ and $\Omega^{\mathrm{in}}$. More precisely, we denote by $\Omega_{h}^{\mathrm{ex}}$ and $\Omega_{h}^{\text {in }}$ the domains covered by the mesh elements of $\mathscr{T}^{\text {ex }}$ and $\mathscr{T}^{\text {in }}$, resp., and we only require that $\Omega^{\mathrm{ex}} \subset \Omega_{h}^{\mathrm{ex}} \subset \Omega, \Gamma \subset \partial \Omega^{\mathrm{ex}}$ and $\Omega^{\text {in }} \subset \Omega_{h}^{\text {in }} \subset \Omega$. Hence the approximation of (2) enters into the framework of overlapping domain decomposition methods. Let $\gamma^{\mathrm{ex}}=\partial \Omega_{h}^{\mathrm{ex}} \backslash \Gamma$ and $\gamma^{\text {in }}=\partial \Omega_{h}^{\text {in }}$ be the two boundaries of $\Omega_{h}^{\mathrm{ex}}$ and $\Omega_{h}^{\text {in }}$ in $\Omega$ that replace the interface $\gamma$. Then we introduce the space

$$
\mathscr{V}_{g}=\left\{(v, w) \in \mathscr{V}^{\mathrm{ex}} \times \mathscr{V}^{\mathrm{in}}, v_{\mid \Gamma}=\Pi^{\mathrm{Dir}} g, v_{\mid \gamma^{\mathrm{ex}}}=\Pi^{\mathrm{ex}} w, w_{\mid \gamma^{\mathrm{in}}}=\Pi^{\mathrm{in}} v\right\},
$$

where $\mathscr{V}^{\text {ex }}$ and $\mathscr{V}^{\text {in }}$ are $H^{1}\left(\Omega_{h}^{\mathrm{ex}}\right)$ and $H^{1}\left(\Omega_{h}^{\text {in }}\right)$ conforming FE spaces defined over $\mathscr{T}^{\mathrm{ex}}$ and $\mathscr{T}^{\text {in }}$. The operators $\Pi^{\mathrm{Dir}}, \Pi^{\mathrm{ex}}$ and $\Pi^{\text {in }}$ are projections onto the Dirichlet trace spaces $\mathscr{V}_{\Gamma}=\operatorname{tr}_{\mid \Gamma} \mathscr{V}^{\mathrm{ex}}, \mathscr{V}_{\gamma}^{\mathrm{ex}}:=\operatorname{tr}_{\mid \gamma^{\mathrm{ex}}} \mathscr{V}^{\mathrm{ex}}$ and $\mathscr{V}_{\gamma}^{\text {in }}:=\operatorname{tr}_{\mid \gamma^{\text {in }}} \mathscr{V}^{\text {in }}$. The MEM with overlapping domains $[10,3,1]$ applied to (2) reads: Find $\left(\psi^{\mathrm{ex}}, \psi^{\mathrm{in}}\right) \in \mathscr{V}_{\psi_{0}}$ such that

$$
\mathrm{a}_{s}^{\mathrm{ex}}\left(\psi^{\mathrm{ex}}, v\right)+\mathrm{a}_{t}^{\mathrm{in}}\left(\psi^{\mathrm{in}}, w\right)=\ell_{s}^{\mathrm{ex}}(f, v)+\ell_{t}^{\mathrm{in}}(f, w) \quad \forall(v, w) \in \mathscr{V}_{0},
$$

where

$$
\begin{aligned}
\mathrm{a}_{s}^{\mathrm{M}}(\psi, v) & :=\int_{\Omega_{h}^{\mathrm{M}}} \nabla \psi \cdot \nabla v \mathrm{~d} \mathbf{x}-\int_{\Omega_{h}^{\mathrm{ex}} \cap \Omega_{h}^{\mathrm{in}}} s \nabla \psi \cdot \nabla v \mathrm{~d} \mathbf{x}, \\
\ell_{s}^{\mathrm{M}}(f, v) & :=\int_{\Omega_{h}^{\mathrm{M}}} f v \mathrm{~d} \mathbf{x}-\int_{\Omega_{h}^{\mathrm{ex}} \cap \Omega_{h}^{\mathrm{in}}} s f v \mathrm{~d} \mathbf{x},
\end{aligned}
$$

for $\mathrm{M}=$ ex and $\mathrm{M}=$ in. Optimal convergence results are available when $s+t=1$ and $\Pi^{\text {ex }}, \Pi^{\text {in }}$, are the $L^{2}$ projections onto $\operatorname{tr}_{\mid \gamma^{\text {ex }}} \mathscr{V}^{\text {ex }}, \operatorname{tr}_{\mid \gamma_{\text {in }}} \mathscr{V}^{\text {in }}$, resp. [3, 1]. However, two very restrictive disadvantages occur with the formulation (3):

1. The assembling of the stiffness matrices associated to $a_{s}^{\mathrm{ex}}(\cdot, \cdot)$ and $\mathrm{a}_{t}^{\mathrm{in}}(\cdot, \cdot)$ involves products of basis functions defined on different meshes. Similarly, the assembling of the load vectors corresponding to $\ell_{s}^{\mathrm{ex}}(f, \cdot)$ and $\ell_{t}^{\mathrm{in}}(f, \cdot)$ involves integration over intersections of elements from different meshes.

2. The stability of MEMs requires the projections $\Pi^{\text {ex }}$ and $\Pi^{\text {in }}$ to be stable in $H^{\frac{1}{2}}$. The obvious choice of $L^{2}$ projections involves again surface integrals of products of basis functions defined on different meshes.

In the following section we will introduce two mortar-like mappings different from the standard $L^{2}$ projection, that allow to choose $s=t=0$ in (3) and hence avoid the assembling of the stiffness matrix for basis functions on two different meshes. 


\section{A Modified Mortar Mapping (MEM-M)}

We recall that the FE spaces $\mathscr{V}^{\mathrm{ex}}$ and $\mathscr{V}^{\text {in }}$ can be represented as direct sums $\mathscr{V}^{\mathrm{ex}}=$ $\mathscr{V}_{0}^{\text {ex }} \oplus \mathscr{V}_{\gamma}^{\text {ex }}$ and $\mathscr{V}^{\text {in }}=\mathscr{V}_{\circ}^{\text {in }} \oplus \mathscr{V}_{\gamma}^{\text {in }}$ where $\mathscr{V}_{\gamma}^{\text {ex }}=\operatorname{tr}_{\mid \gamma^{\text {ex }}} \mathscr{V}^{\text {ex }}$ and $\mathscr{V}_{\gamma}^{\text {in }}=\operatorname{tr}_{\mid \gamma^{\text {in }}} \mathscr{V}^{\text {in }}$ are the earlier introduced trace spaces of $\mathscr{V}^{\mathrm{ex}}$ and $\mathscr{V}^{\mathrm{in}}$. Let us define two mappings $\Pi_{f}^{\mathrm{ex}} \psi^{\mathrm{in}}$ for $\psi^{\text {in }}=\psi_{\circ}^{\text {in }}+\psi_{\gamma}^{\text {in }}$, with $\psi_{\circ}^{\text {in }} \in \mathscr{V}_{\circ}^{\text {in }}, \psi_{\gamma}^{\text {in }} \in \mathscr{V}_{\gamma}^{\text {in }}$ and $\Pi_{f}^{\text {in }} \psi^{\text {ex }}$ for $\psi^{\mathrm{ex}}=\psi_{\circ}^{\mathrm{ex}}+\psi_{\gamma}^{\mathrm{ex}}$, with $\psi_{\circ}^{\mathrm{ex}} \in \mathscr{V}_{\circ}^{\mathrm{ex}}, \psi_{\gamma}^{\mathrm{ex}} \in \mathscr{V}_{\gamma}^{\mathrm{ex}}$ with:

$$
\Pi_{f}^{\mathrm{ex}} \psi^{\text {in }}:=\Pi^{\mathrm{ex}}\left(\psi_{\gamma}^{\text {in }}+\Psi_{\circ}^{\text {in }}\right), \text { with } \mathrm{a}_{0}^{\text {in }}\left(\Psi_{\circ}^{\text {in }}, w\right)=\ell_{0}^{\text {in }}(f, w)-\mathrm{a}_{0}^{\text {in }}\left(\psi_{\gamma}^{\text {in }}, w\right) \forall w \in \mathscr{V}_{\circ}^{\text {in }},
$$

where $\Pi^{\mathrm{ex}}$ is either the $L^{2}$-projection or standard nodal interpolation operator onto $\mathscr{V}_{\gamma}^{\mathrm{ex}}$ and $\Pi_{f}^{\mathrm{in}}$ analogously defined. We then introduce the space

$$
\mathscr{V}_{g, f}=\left\{(v, w) \in \mathscr{V}^{\mathrm{ex}} \times \mathscr{V}^{\mathrm{in}}, v_{\mid \Gamma}=\Pi^{\mathrm{Dir}} g, v_{\mid \gamma^{\mathrm{ex}}}=\Pi_{f}^{\mathrm{ex}} w, w_{\mid \gamma^{\mathrm{in}}}=\Pi_{f}^{\mathrm{in}} v\right\}
$$

and obtain the following modified version of the MEM for overlapping meshes: Find $\left(\psi^{\mathrm{ex}}, \psi^{\mathrm{in}}\right) \in \mathscr{V}_{\psi_{0}, f}$ such that

$$
\mathrm{a}_{0}^{\mathrm{ex}}\left(\psi^{\mathrm{ex}}, v\right)+\mathrm{a}_{0}^{\mathrm{in}}\left(\psi^{\mathrm{in}}, w\right)=\ell_{0}^{\mathrm{ex}}(f, v)+\ell_{0}^{\mathrm{in}}(f, w) \quad \forall(v, w) \in \mathscr{V}_{0,0},
$$

A similar approach with the lowest order FE spaces in the non-destructive testing context has been adopted in [5, 6]. It can be shown that (4) is equivalent to the following formulation. Find $\left(\psi^{\mathrm{ex}}, \psi^{\mathrm{in}}\right) \in \mathscr{V}^{\mathrm{ex}} \times \mathscr{V}^{\mathrm{in}}, \psi_{\mid \Gamma}^{\mathrm{ex}}=\Pi^{\mathrm{Dir}} g$ such that:

$$
\begin{aligned}
& \mathrm{a}_{0}^{\mathrm{ex}}\left(\psi^{\mathrm{ex}}, v\right)+\mathrm{a}_{0}^{\mathrm{in}}\left(\psi^{\mathrm{in}}, w\right)=\ell_{0}^{\mathrm{ex}}(f, v)+\ell_{0}^{\mathrm{in}}(f, w) \quad \forall(v, w) \in \mathscr{V}_{\circ}^{\mathrm{ex}} \times \mathscr{V}_{\circ}^{\text {in }}, v_{\mid \Gamma=0} \\
& \psi_{\mid \gamma^{\mathrm{ex}}}^{\mathrm{ex}}=\Pi^{\mathrm{in}} \psi^{\text {in }}, \quad \psi_{\mid \gamma^{\text {in }}}^{\text {in }}=\Pi^{\mathrm{ex}} \psi^{\mathrm{ex}},
\end{aligned}
$$

which corresponds to the numerical zoom formulation in [8]. When the $\Pi^{\mathrm{ex}}$ and $\Pi^{\text {in }}$ are interpolation operators and $\mathscr{V}^{\mathrm{ex}}$ and $\mathscr{V}^{\mathrm{in}}$ are lowest order Lagrangian FE spaces we can recall an optimal convergence result from [11, Theorem 1] for the error in the $L^{\infty}$-norm.

\section{Numerical Experiments}

For the numerical experiments, we consider a rectangular domain $\Omega=[-1,1]^{2}$ and define $\Omega^{\text {in }}$ as the polygon with vertices $(-0.125,0.5),(0.375,0.25),(0.375,-0.375)$, $(0,-0.5),(-0.375,-0.375)$, and $(-0.5,0.25)$. The meshes $\mathscr{T}^{\text {in }}$ and $\mathscr{T}^{\text {ex }}$ for the interior and exterior domain will be a Cartesian mesh and a triangular mesh. For simplicity we prefer to take $\Omega_{h}^{\mathrm{ex}}=\Omega^{\mathrm{ex}}=\Omega \backslash \Omega^{\mathrm{in}}$. For the numerical test, we choose the data $f(x, y)$ and $\psi_{0}$ such that $\psi(x, y)=\cos (\pi x) \sin (\pi y)$ is the solution of (1). If $h_{\mathrm{ex}}$ (resp. $h_{\text {in }}$ ) is the maximal diameter of elements in $\mathscr{T}^{\text {ex }}\left(\right.$ resp. $\mathscr{T}^{\text {in }}$ ), and $p_{\text {ex }}$ (resp. $\left.p_{\text {in }}\right)$ the local polynomial degree of the FE spaces $\mathscr{V}^{\text {ex }}$ (resp. $\mathscr{V}^{\text {in }}$ ), one has optimal 
convergence if, for a smooth solution, the approximation error in the $H^{1}\left(\Omega_{h}^{\mathrm{ex}}\right)$ and $H^{1}\left(\Omega_{h}^{\text {in }}\right)$-norms behaves as $O\left(h^{p-1}\right)$, with $h=\max \left(h_{\mathrm{ex}}, h_{\text {in }}\right)$ and $p=\min \left(p_{\mathrm{ex}}, p_{\text {in }}\right)$ (in $L^{2}\left(\Omega_{h}^{\text {ex }}\right)$ and $L^{2}\left(\Omega_{h}^{\text {in }}\right)$-norms one dares to obtain $O\left(h^{p}\right)$ ). To keep the presentation as clear as possible we show in the following figures always the maximum between the error in $\Omega_{h}^{\text {ex }}$ and that in $\Omega_{h}^{\text {in }}$.
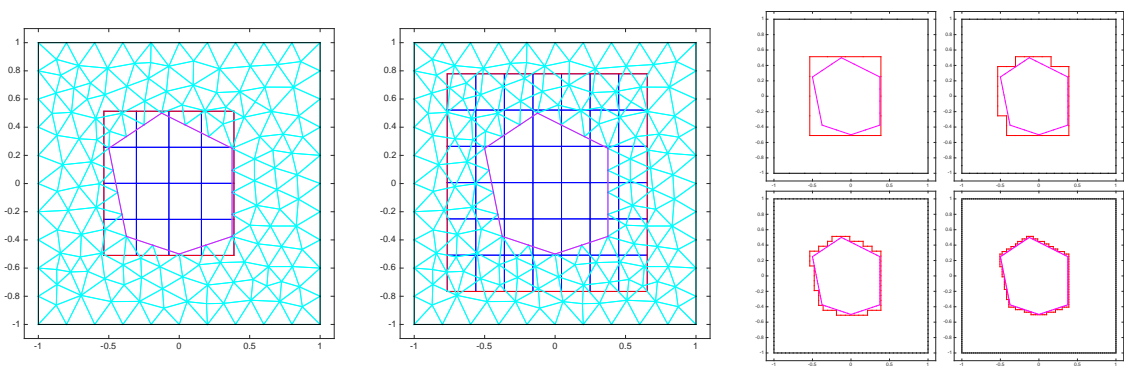

Fig. 3 Left and Center: Sketch of the two different settings. We may choose $\Omega_{h}^{\text {in }}$ to have a minimal overlap with $\Omega_{h}^{\text {ex }}$ (left), that is $\gamma^{\text {ex }}$ is contained in the layer of elements of $\mathscr{T}^{\text {in }}$ which define $\gamma^{\text {in }}$. Otherwise, we say that $\Omega_{h}^{\text {in }}$ has a large overlap with $\Omega_{h}^{\text {ex }}$ (center). Right: Adaptive definition of $\gamma^{\text {in }}$ in the case of minimal overlap between $\Omega_{h}^{\text {in }}$ and $\Omega_{h}^{\mathrm{ex}}$. Note that, $\gamma^{\mathrm{ex}}$ (magenta) remains fixed, while $\gamma^{\text {in }}$ (red) changes due to the refinements in $\Omega_{h}^{\text {in }}$. The interior edges of elements of $\mathscr{T}^{\text {ex }}$ and $\mathscr{T}^{\text {in }}$ are omitted for clearness.

We consider two different pairings of FE spaces $\mathscr{V}^{\mathrm{ex}} \mathscr{V}^{\mathrm{in}}$. The first denoted with P1-Q1 uses lowest order linear FEs over $\mathscr{T}^{\mathrm{ex}}$ and lowest order bilinear FEs over $\mathscr{T}^{\text {in }}$. The second pair, denoted with P2-Q3 uses quadratic FEs over $\mathscr{T}^{\text {ex }}$ and bicubic FEs over $\mathscr{T}^{\text {in }}$. The elements of P2-Q3 are not only continuous on $\Omega_{h}^{\text {in }}$ and $\Omega_{h}^{\text {ex }}$ but have also continuous gradients on $\Omega_{h}^{\text {in }}$.
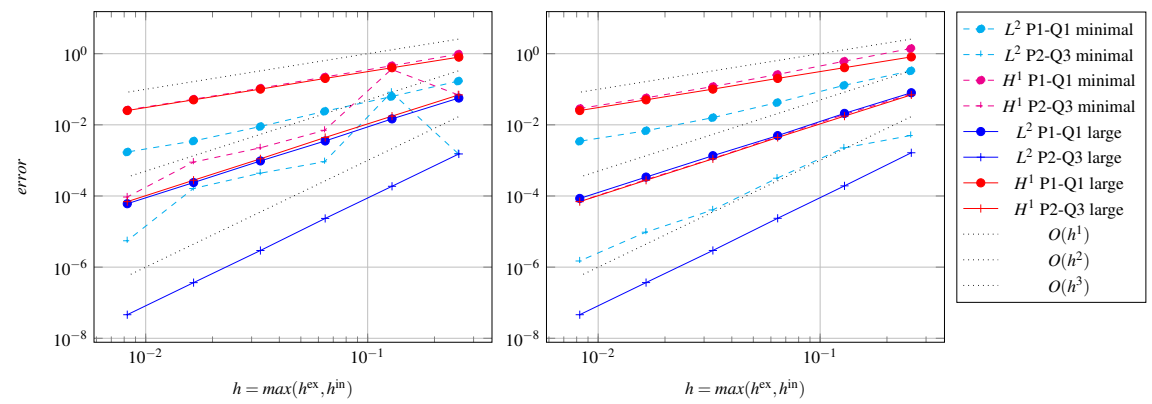

Fig. 4 Convergence in $L^{2}$ and $H^{1}$ of the scheme MEM-M using $L^{2}$-projection (left) or nodal interpolation (right).

We focus on the overlapping MEM-M (4) which uses the modified mortar mappings and is equivalent to (5). We also analyse the influence on the error curves of 
using either $L^{2}$ projections or interpolation to realize the gluing across $\gamma^{\mathrm{ex}}$ and $\gamma^{\text {in }}$ for the MEM-M. We start with the case where $\Omega_{h}^{\text {in }}$ has minimal overlap with $\Omega_{h}^{\text {ex }}$ (see Fig. 3, left). Thus $\gamma^{\text {in }}$ is adapted with the refinements in $\Omega_{h}^{\text {in }}$ as shown in Fig. 3. Convergence results with MEM-M are presented in Fig. 4. The convergence rate with MEM-M is optimal for the error in the $H^{1}$-norm. The results look slightly better if we apply the interpolation instead of the $L^{2}$ projection in the definition of the mortar mapping.

Next, we study the convergence rates for MEM-M when $\Omega_{h}^{\text {in }}$ has a large overlap with $\Omega_{h}^{\text {ex }}$ (see Fig. 3, right). Note that both $\gamma^{\text {ex }}$ and $\gamma^{\text {in }}$ remain fixed during the refinements in $\Omega_{h}^{\text {in }}$. Once again, the MEM-M yields optimal convergence rate in the $H^{1}$ norm (see Fig. 4). Moreover in the case of larger overlap we observe even optimal convergence in the $L^{2}$-norm. There is no qualitative difference between MEM-M based on the $L^{2}$-projection or on the interpolation.

With the classical overlapping MEM (3) with the parameters $s$ and $t$ set to zero (MEM-0), the convergence rates in the $H^{1}$ and $L^{2}$ norms are not optimal in the case of minimal overlap between $\Omega_{h}^{\text {in }}$ and $\Omega_{h}^{\text {ex }}$. The MEM-0 does not yield convergence in the case of a large overlap between $\Omega_{h}^{\text {in }}$ and $\Omega_{h}^{\text {ex }}$.

\section{References}

1. Y. Achdou, Y. Maday. The mortar element method with overlapping subdomains. SIAM J. Numer. Anal., 40(2):601-628, 2002.

2. J. Blum. Numerical Simulation and Optimal Control in Plasma Physics. Wiley/GauthierVillars Series in Modern Applied Mathematics, 1989.

3. X.-C. Cai, M. Dryja, M. Sarkis. Overlapping nonmatching grid mortar element methods for elliptic problems. SIAM J. Numer. Anal., 36(2):581-606, 1999.

4. G. Chesshire, W.D. Henshaw. Composite overlapping meshes for the solution of partial differential equations. J. Comput. Phys., 90(1):1-64, 1990.

5. A. Christophe, L. Santandrea, F. Rapetti, G. Krebs, Y. Le Bihan. An overlapping non-matching grid mortar element method for Maxwell's equations. IEEE Trans. Magn., 50(2), 2014.

6. A. Christophe, Y. Le Bihan, F. Rapetti. A mortar element approach on overlapping non-nested grids: application to eddy current non-destructive testing. Appl. Math. Comp., 267:71-82, 2015.

7. J. Fish, T. Belytschko. Elements with embedded localization zones for large deformation problems. Comput. \& Struct., 30(1):247-256, 1988.

8. F. Hecht, A. Lozinski, O. Pironneau. Numerical zoom and the Schwarz algorithm. In Domain decomposition methods in science and engineering XVIII, LNCSE 70:63-73, Springer, Berlin, 2009.

9. H. Heumann, F. Rapetti. A finite element method with overlapping meshes for free-boundary axisymmetric plasma equilibria in realistic geometries. RR-8916, Inria SAM, 2016.

10. Y. A. Kuznetsov. Overlapping domain decomposition with non-matching grids. In Recent developments in domain decomposition methods and flow problems (Kyoto, 1996; Anacapri, 1996), volume 11 of GAKUTO Int. Ser. Math. Sci. Appl., 62-71. Gakkōtosho, Tokyo, 1998.

11. A. Lozinski, O. Pironneau. Numerical zoom for advection diffusion problems with localized multiscales. Numer. Meth. PDEs, 27(1):197-207, 2011.

12. C. D. Mote, Jr. Global-local finite element. Int. J. Numer. Meth. Engrg., 3:565-574, 1971.

13. S. V. Parter. On the overlapping grid method for elliptic boundary value problems. SIAM J. Numer. Anal., 36(3):819-852, 1999.

14. J. L. Steger, J. A. Benek. On the use of composite grid schemes in computational aerodynamics. In Procs of the first world congress on computational mechanics, Austin, Tex., 1986, 64:301-320, 1987. 